\title{
The Use of Adaptive Statistical Iterative Reconstruction in Pediatric Head CT: A Feasibility Study
}

\author{
G.A. Vorona, G. Zuccoli, T. Sutcavage, B.L. Clayton, R.C. Ceschin, and A. Panigrahy
}

\begin{abstract}
BACKGROUND AND PURPOSE: Iterative reconstruction techniques facilitate CT dose reduction; though to our knowledge, no group has explored using iterative reconstruction with pediatric head CT. Our purpose was to perform a feasibility study to assess the use of ASIR in a small group of pediatric patients undergoing head $\mathrm{CT}$.
\end{abstract}

MATERIALS AND METHODS: An Alderson-Rando head phantom was scanned at decreasing 10\% $\mathrm{mA}$ intervals relative to our standard protocol, and each study was then reconstructed at 10\% ASIR intervals. An intracranial region of interest was consistently placed to estimate noise. Our ventriculoperitoneal shunt CT protocol was subsequently modified, and patients were scanned at $20 \%$ ASIR with approximately $20 \% \mathrm{~mA}$ reductions. ASIR studies were anonymously compared with older non-ASIR studies from the same patients by 2 attending pediatric neuroradiologists for diagnostic utility, sharpness, noise, and artifacts.

RESULTS: The phantom study demonstrated similar noise at 100\% mA/0\% ASIR (3.9) and $80 \% \mathrm{~mA} / 20 \%$ ASIR (3.7). Twelve pediatric patients were scanned at reduced dose at $20 \%$ ASIR. The average $\mathrm{CTDI}_{\text {vol }}$ and DLP values of the $20 \%$ ASIR studies were $22.4 \mathrm{mGy}$ and $338.4 \mathrm{mGy}$ - $\mathrm{cm}$, and for the non-ASIR studies, they were $28.8 \mathrm{mGy}$ and $444.5 \mathrm{mGy}-\mathrm{cm}$, representing statistically significant decreases in the CTDI vol $^{2}(22.1 \%$, $P=.00007)$ and DLP $(23.9 \%, P=.0005)$ values. There were no significant differences between the ASIR studies and non-ASIR studies with respect to diagnostic acceptability, sharpness, noise, or artifacts.

CONCLUSIONS: Our findings suggest that $20 \%$ ASIR can provide approximately $22 \%$ dose reduction in pediatric head CT without affecting image quality.

ABBREVIATIONS: $\mathrm{ACR}=$ American College of Radiology; $\mathrm{ASIR}=$ adaptive statistical iterative reconstruction; $\mathrm{CNR}=$ contrast-to-noise ratio; $\mathrm{CTDI}=\mathrm{CT}$ dose index; $\mathrm{CTDI}_{\text {vol }}=\mathrm{CT}$ dose index volume; $\mathrm{DLP}=$ dose-length product; $\mathrm{FBP}=$ filtered back-projection; $\mathrm{IR}=$ iterative reconstruction; IRIS = iterative reconstruction in image space

O the approximately 70 million CT examinations performed in this country in $2007,{ }^{1}$ it is estimated that approximately $7 \%$ (4.9 million) of these were performed in persons younger than 18 years of age. ${ }^{2}$ The potential carcinogenic effects of ionizing radiation inherent to CT are particularly concerning in the pedi-

Received January 5, 2012; accepted after revision January 25.

From the Department of Radiology (G.A.V.), Allegheny General Hospital, Pittsburgh, Pennsylvania; and Children's Hospital of Pittsburgh of the University of Pittsburgh Medical Center (G.Z., T.S., B.L.C., R.C.C., A.P.), Pittsburgh, Pennsylvania.

G.A.V., G.Z., T.S., B.L.C., and R.C.C. have no pertinent disclosures and had full control of the study data during the study.

No grant support was received for this research.

Previously presented at: Annual Meeting of the Society of Pediatric Radiology, April 16-20, 2012; San Francisco, California.

Please address correspondence to Ashok Panigrahy, MD, Radiologist-in-Chief, Associate Professor of Radiology, Children's Hospital of Pittsburgh of University of Pittsburgh Medical Center, Department of Radiology, 4401 Penn Avenue, Pittsburgh, PA 15224; e-mail: panigrahya@upmc.edu

http://dx.doi.org/10.3174/ajnr.A3122 atric age group because children's tissues are inherently more radiosensitive and because children have a longer lifetime to manifest radiation-induced injury. ${ }^{3}$ For these reasons, the Alliance for Radiation Safety in Pediatric Imaging has worked to minimize unnecessary radiation doses for children, particularly through their Image Gently educational and awareness campaign. ${ }^{4}$

A recent study examining the use of CT in child visits to the emergency department in the United States from 1995 to 2008 found a substantial and sustained growth in the use of CT during this time. The 2 most commonly recorded conditions for visits in which CT scanning was performed were "head injury" followed by "headache". ${ }^{5}$ Although the brain is generally considered to be more radio-resistant than other body tissues, data show that low doses of radiation to this region can significantly contribute to future cancer development in children. ${ }^{6}$ Brenner et $\mathrm{al}^{7}$ estimated a lifetime mortality risk of $0.07 \%$ from a single pediatric head CT in a 1-year-old child. Additional research indicates that a 3-yearold boy has approximately 9 times the risk of developing cancer as 
a result of a single head CT examination compared with an older adult. ${ }^{2}$ The deterministic effects of ionizing radiation should also be considered. A study following pediatric patients who underwent repeated head CT examinations after cholesteatoma resection found that these patients received a level of radiation dose to the lens approximating the epidemiologic dose necessary for the development of lens opacities. ${ }^{8}$

Advances in computers have facilitated the development of hardware capable of the computational power needed for the practical use of IR, and IR techniques are becoming increasingly used in the clinical setting. All of the major CT manufacturers have recently introduced IR algorithms for their respective CT units, which include ASIR (GE Healthcare, Milwaukee, Wisconsin), IRIS (Siemens, Erlangen, Germany), Adaptive Iterative Dose Reduction (Toshiba, Tochigi, Japan), and iDose (Philips Healthcare, Best, the Netherlands). Essentially, these are all "trial and error" reconstruction methodologies that go through several iterations to create an "ideal image" from the obtained projection data, by using a statistical model of noise to improve the image quality with each successive iteration. A major advantage of these techniques is the enhanced image quality compared with routine FBP in the presence of a lower SNR. In addition to noise, the newest IR techniques (eg, Model-Based Iterative Reconstruction, Veo; GE Healthcare) are able to model the geometry of the CT unit itself but are currently limited in applicability by their substantially longer computational times. ${ }^{9}$ Multiple studies have been performed in an attempt to validate the use of ASIR in imaging the chest, ${ }^{10,11}$ abdomen, ${ }^{12-14}$ heart, ${ }^{15-17}$ and vascular structures. ${ }^{18}$ At the time of this writing, only 2 publications have explored the use of IR techniques in pediatric imaging, ${ }^{19,20}$ both having used ASIR.

There has been some interest in incorporating IR techniques into head CT protocols, because theoretically, lower radiation doses could be used without impacting image quality. Recent publications have explored the use of IR in adult head CT with both ASIR $^{21}$ and IRIS, ${ }^{22}$ and these studies suggest that a $20 \%-$ $30 \%$ dose reduction in head CT is feasible in adults by using these iterative techniques with preservation of image quality. To our knowledge, no studies have been performed to assess the potential use of iterative reconstruction techniques to reduce pediatric head CT dose. The purpose of our study was to establish a pediatric head CT protocol using ASIR through phantom testing and to assess the feasibility of this protocol through scanning a pediatric patient population undergoing repeat head CT examinations.

\section{MATERIALS AND METHODS Phantom Testing}

The head of an Alderson-Rando small female phantom (Radiology Support Devices, Long Beach, California) was obtained from the radiation oncology department of our institution. This phantom contains a human skull molded in tissue-equivalent material. A scout topogram of this phantom was obtained on our departmental LightSpeed VCT 64-MDCT unit (GE Healthcare). An anteroposterior measurement was obtained by using the distance between the glabella and opisthocranion $(19.7 \mathrm{~cm})$, and a transverse diameter was obtained by using the maximal parietal diameter $(14.2 \mathrm{~cm})$. With the data published by Kleinman et $\mathrm{al}^{23}$ the phantom was found to best approximate the size of a 7-year-old child's head.

Our institution uses an age-based head CT protocol based on vendor recommendations and the experience of the faculty. The Rando phantom was scanned with identical scan parameters (milliampere, kilovolt[peak], section thickness, and so forth) as those used in a 7-year-old child ( $150 \mathrm{~mA}, 120 \mathrm{kV}$ [p], axial mode, gantry rotation speed $=800 \mathrm{~ms}, 120 \mathrm{mAs}, 2 \times 5 \mathrm{~mm}$ section thickness) at $0 \%$ ASIR. Subsequently, the phantom was scanned at decreasing $10 \% \mathrm{~mA}$ intervals with all other scan parameters constant, down to and including 50\% $\mathrm{mA}(75 \mathrm{~mA})$. Each study was then reconstructed at increasing $10 \%$ ASIR intervals, from $0 \%$ to $100 \%$. In total, 66 scans of varying $\mathrm{mAs}$ and \%ASIR were reconstructed. A $1.0-\mathrm{cm}^{2}$ region of interest was then drawn in an identical position $1.0 \mathrm{~cm}$ posterior to the midline frontal bone for each study (Fig 1), and the noise (in SDs) was recorded.

As a result of the noise information obtained from the above procedure, a Gammex 464 phantom (Gammex, Middleton, Wisconsin) was scanned with the following CT parameters: full dose (150 mA, 0\% ASIR, $120 \mathrm{kV}[\mathrm{p}]$, axial mode, gantry rotation speed $=800 \mathrm{~ms}, 120 \mathrm{mAs}, 2 \times 5 \mathrm{~mm}$ section thickness) and $80 \%$ dose $(120 \mathrm{~mA}, 20 \%$ ASIR, $120 \mathrm{kV}$ [p], axial mode, gantry rotation speed $=800 \mathrm{~ms}, 96 \mathrm{mAs}, 2 \times 5 \mathrm{~mm}$ section thickness). These CT phantom examinations were assessed for low contrast resolution, uniformity, and artifacts as outlined by the most recent ACR Accreditation Phantom Instructions. ${ }^{24}$ Complete calibration of the phantom was performed according to the ACR specifications immediately before phantom data image acquisition.

\section{Patient Selection and Scanning Technique}

This Health Insurance Portability and Accountability Act-compliant study was approved by the institutional Health Systems Quality Improvement Committee, a subset of the institutional review board. The need for written informed consent was waived.

As a result of our phantom data, the decision was made to reduce the $\mathrm{mA}$ by approximately $20 \%$ for pediatric head CT examinations for the indication of "ventriculoperitoneal shunt follow-up," which were then all reconstructed with $20 \%$ ASIR. All of these prospective studies were performed on the same LightSpeed VCT 64-MDCT unit (GE Healthcare). All other scan parameters besides milliampere were kept consistent compared with our current routine age-based pediatric head CT protocol. From April 1, 2011, to September 1, 2011, 16 patients were prospectively scanned by using this revised protocol. The imaging history of these 16 patients was then reviewed on PACS, with particular attention to prior head CT examinations performed in the preceding few years to create "comparison study sets." Each comparison study set included the ASIR study and an older head CT reconstructed only with FBP. Inclusion criteria for prior FBP examinations to include in each comparison study set were the same kilovolt(peak), same section thickness, the same scan FOV and displayed FOV, and reported dose information. Only nonenhanced head CT studies were included. Exclusion criteria were the use of ASIR and dose information that was not reported.

Before May 1, 2009, our radiology department was performing head CT studies by using a HighSpeed CT/I unit in the axial mode (GE Healthcare), and 3 studies from this unit were included for 

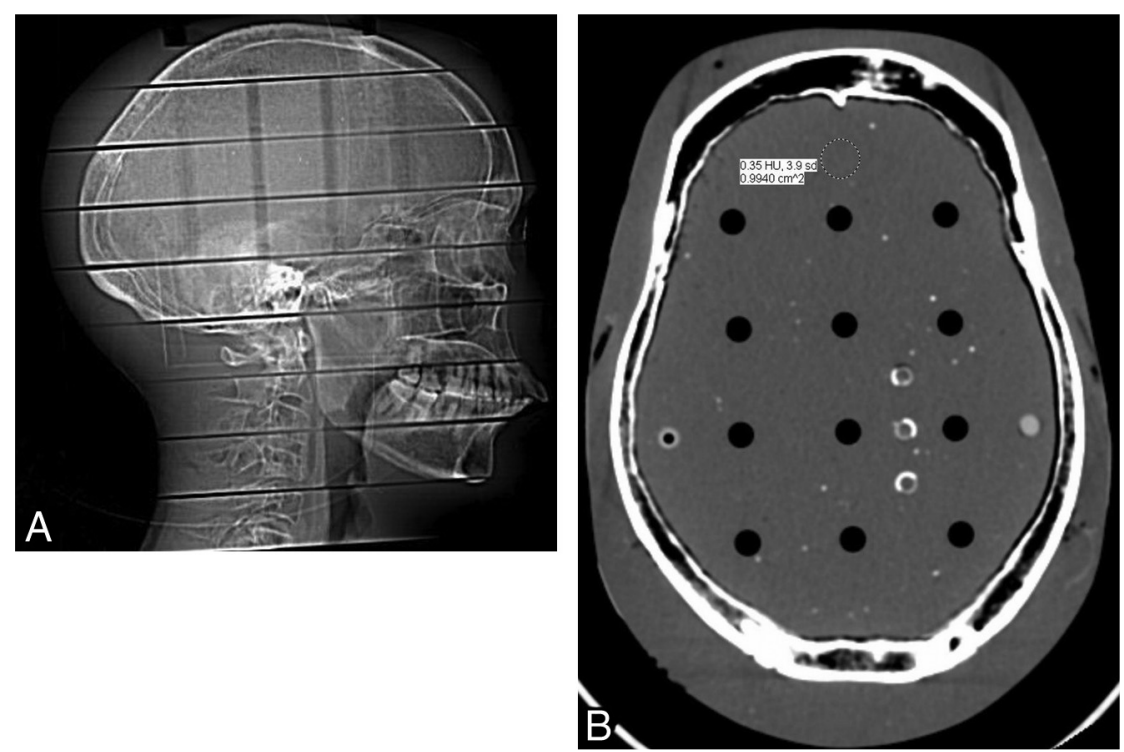

FIG 1. A, Lateral scout film of the Alderson-Rando phantom. $B$, Axial section of the phantom scanned at $150 \mathrm{~mA}$, indicating region-of-interest placement.

comparison. These 3 FBP studies performed on the HighSpeed CT/I unit had identical kilovolt(peak), section thickness, gantry rotation speed, and scan FOV/displayed FOV compared with their corresponding ASIR studies performed on the LightSpeed VCT 64-MDCT. One of the comparison study sets, in which both studies were scanned on the LightSpeed VCT 64-MDCT, had minimally different gantry rotation speeds ( $900 \mathrm{~ms}$ for the ASIR study, $800 \mathrm{~ms}$ for the older FBP study). Otherwise, all comparison study sets had identical gantry rotation speeds. Twelve comparison study sets were identified by using these criteria ( 6 males and 5 females; mean age, 9 years at time of ASIR study; range, 3-18 years). The average amount of time between the ASIR and FBP studies was 349 days, with a range of 27-871 days. Every patient had a ventriculoperitoneal shunt catheter present in the same location on both their ASIR and FBP CT examinations. No study used automatic tube-current modulation.

\section{Radiation Dose Measurements}

The CTDI ${ }_{\mathrm{vol}}$ and DLP values were obtained for each of the ASIR and FBP studies by using the PACS of this institution. The accuracy of the displayed $\mathrm{CTDI}_{\mathrm{vol}}$ and DLP of the manufacturer was regularly tested as a part of the quality control program of this institution.

\section{Quantitative Measurements}

For each examination, a $0.5-1.0 \mathrm{~cm}^{2}$ region of interest was drawn to measure noise (SD) in the air, WM, bone, and CSF. There was consistent placement and sizing of regions of interest between the ASIR and FBP CT studies in a comparison study set, and the decision of where to place the region of interest for a given attenuation was made on the basis of the largest homogeneous area to sample of that density. The region of interest in air was drawn 1.0 $\mathrm{cm}$ lateral to the superior left orbit. The region of interest in WM was drawn in the parietal white matter. The region of interest for bone was obtained either in the region of the planum sphenoidale or the greater wing of the sphenoid. The region of interest for CSF was placed either in the lateral ventricle or the fourth ventricle.

\section{Phantom Study}

\section{Qualitative Measurements}

Qualitative analysis was performed by anonymizing each ASIR and FBP study, including removing all displayed technical and personal medical information. Twenty-four head CT studies were included in all. These studies were then randomly displayed on a high-resolution diagnostic monitor, the "brain window" $(80 / 35)$ of our institution, and were independently evaluated by 2 attending pediatric neuroradiologists with $>10$ years of combined experience. The radiologists were neither informed about whether a given study was reconstructed with or without ASIR nor how many ASIR and FBP studies were in the study set.

The scale that we used to assess subjective image quality was based from the scale used by Kilic et $\mathrm{al}^{21}$ in their subjective analysis. Subjective image noise was assessed on a 4-point scale: $1=$ least noise, too little noise (below average noise); 2 = optimum noise (average noise); 3 = noisy but permits evaluation (above average noise); and $4=$ too much noise so that no information can be gathered. Subjective image sharpness was assessed on a 5-point scale: 1 = structures are well-defined with sharp contours (aboveaverage sharpness); 2 = contours are not fully sharp but structures are defined (average sharpness); $3=$ structures can be seen and contours are barely sharp enough (below average sharpness); $4=$ though structures can be visualized, contours are blurred and images are insufficient for diagnostic reporting; and $5=$ structures cannot be identified. The readers were instructed to evaluate subjective image sharpness by the gray matter and WM differentiation, visualization of the basal ganglia/pons/ventricular system, and visualization of the boundary of the CSF spaces around the mesencephalon and over the brain. Diagnostic acceptability was assessed on a 4 -point scale: $1=$ fully acceptable, $2=$ probably acceptable, $3=$ only acceptable under limited conditions, and $4=$ unacceptable. Artifacts were evaluated on a 4-point scale: $1=$ no artifacts, $2=$ minor artifacts, $3=$ major artifacts but interpretable, and $4=$ artifacts make image interpretation impossible.

\section{Statistical Analysis}

Quantitative image-noise measurements were compared by using the paired Student $t$ test. Interobserver variability was assessed by using the weighted $\kappa$ test. The scores for diagnostic acceptability, sharpness, noise level, and artifacts were averaged across both readers, and these values were compared between the ASIR and FBP studies by using the paired Student $t$ test. $P<.05$ was considered to indicate a statistically significant difference.

\section{RESULTS}

Results by using the Alderson-Rando phantom demonstrated linear decreases in noise, as measured in SDs, when studies using the same tube current were reconstructed with increasing percentages 


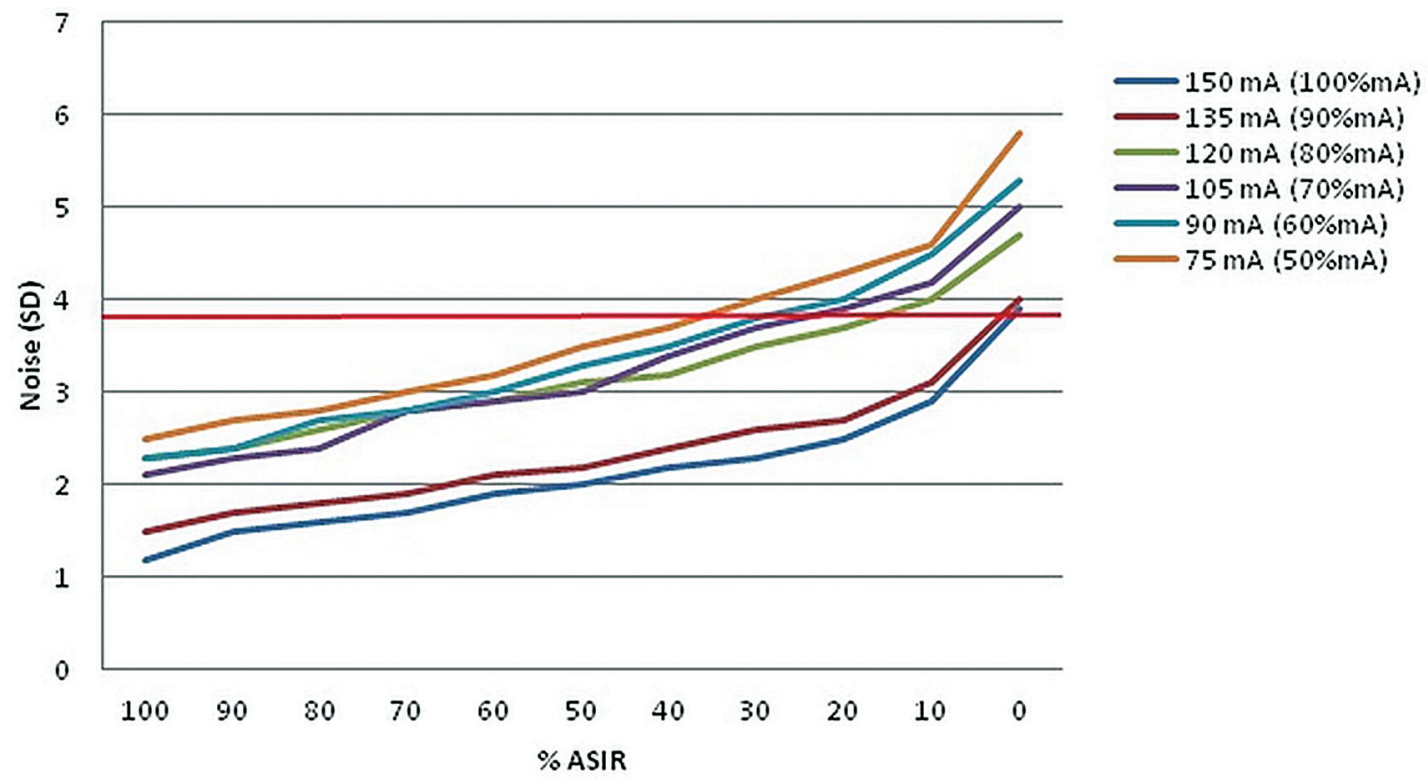

FIG 2. Noise data (SD) scanning of the Rando head phantom at intervals of decreasing milliampere and increasing \% ASIR relative to our age-based institutional settings for a 7-year-old patient. The phantom study suggests similar noise levels at $100 \% \mathrm{~mA} / 0 \% \mathrm{ASIR}$ and $80 \% \mathrm{~mA} / 20 \%$ ASIR (red line).
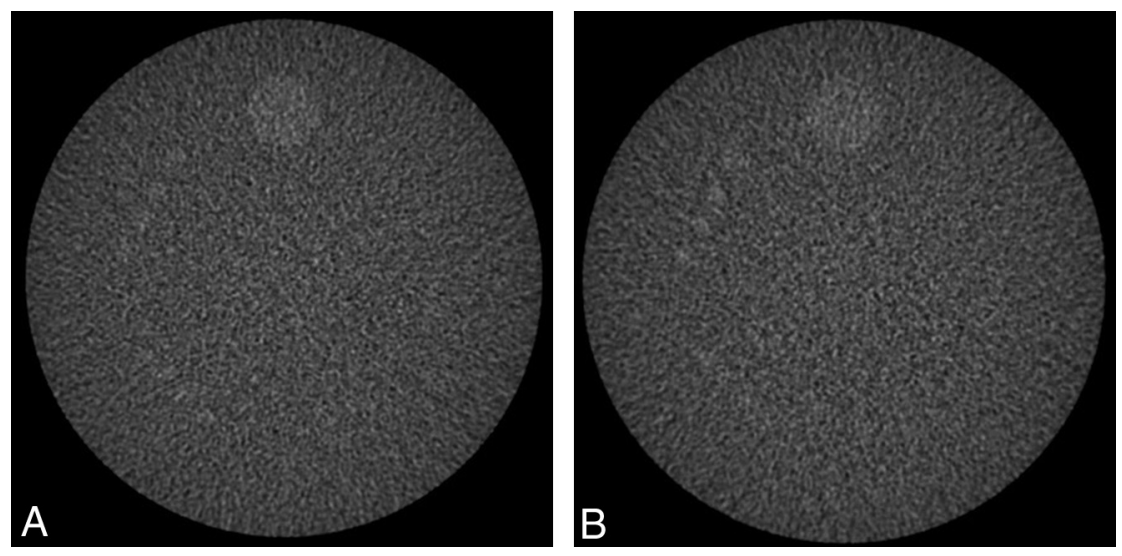

FIG 3. Low-contrast resolution testing of the Gammex 464 phantom at $150 \mathrm{~mA}$ and full FBP $(A)$ and at $120 \mathrm{~mA}$ and $20 \%$ ASIR (B). Both images are set at 100/100 as per ACR guidelines. There is similar visualization of the 6-mm cylinders in both image sets.

Subjective image quality scores ${ }^{\mathrm{a}}$

\begin{tabular}{lcccc}
\hline & $\begin{array}{c}\text { Diagnostic } \\
\text { Acceptability }\end{array}$ & Sharpness & Noise & Artifacts \\
\hline FBP & $1.3 / 1.3 / 1.3$ & $2.2 / 2.2 / 2.2$ & $2.2 / 2.3 / 2.2$ & $2.1 / 2.1 / 2.1$ \\
$20 \%$ ASIR & $1.3 / 1.3 / 1.3$ & $2.0 / 2.1 / 2.0$ & $2.3 / 2.3 / 2.3$ & $1.8 / 1.8 / 1.8$ \\
$P$ value & .33 & .45 & .84 & .06 \\
\hline
\end{tabular}

${ }^{a}$ Listed as reviewer $1 /$ reviewer 2 /average reviewer scores.

of ASIR (Fig 2). The noise at $80 \% \mathrm{~mA}(120 \mathrm{~mA})$ reconstructed at $20 \%$ ASIR was 3.7, almost the equivalent to that of a full dose ( 150 $\mathrm{mA}$ ) at $0 \% \operatorname{ASIR}(3.9)$.

Comparison of low-contrast images scanned at $150 \mathrm{~mA} / 0 \%$ ASIR and $120 \mathrm{~mA} / 20 \%$ ASIR by using the Gammex 464 phantom showed comparable appearances of the 6-mm objects in both (Fig 3). Both image sets had CNR ratios above the minimum 1.0 established by the ACR for accreditation $(\mathrm{CNR}=1.41$ for both sets of images). Uniformity measurements from the 2 different image sets were both within the ACR specifications. No significant artifacts were seen in either image set. ${ }^{24}$

\section{Radiation Dose Measurements}

The average $\mathrm{CTDI}_{\mathrm{vol}}$ and DLP values of the $20 \%$ ASIR studies were $22.4 \mathrm{mGy}$ (range $=15.5-27.5 \mathrm{mGy}, \mathrm{SD}=4.3 \mathrm{mGy})$ and $338.4 \mathrm{mGy}-\mathrm{cm}$ (range $=247.8-444.9$ $\mathrm{mGy}-\mathrm{cm}, \mathrm{SD}=70.3 \mathrm{mGy}-\mathrm{cm})$, respectively. The average CTDI ${ }_{\mathrm{vol}}$ and DLP values of the FBP studies were $28.8 \mathrm{mGy}$ $($ range $=18.7-39.7 \mathrm{mGy}, \mathrm{SD}=7.2 \mathrm{mGy})$ and $444.5 \mathrm{mGy}-\mathrm{cm}$ (range $=289.4-658.3$ $\mathrm{mGy}-\mathrm{cm}, \mathrm{SD}=135.0 \mathrm{mGy}-\mathrm{cm})$, respectively. This finding represents a statistically significant decrease in the CTDI ${ }_{\mathrm{vol}}$ by $22.1 \%(P=.00007)$ and a statistically significant decrease in the DLP by $23.9 \%$ $(P=.0005)$.

\section{Quantitative Analysis}

The average noise values in the 20\% ASIR studies for air, $\mathrm{WM}$, bone, and CSF were 3.0 (range $=1.9-4.9, \mathrm{SD}=1.0$ ), 4.0 (range $=3.1-5.2, \mathrm{SD}=0.6), 55.2($ range $=21.9-95.9$, $\mathrm{SD}=20.1$ ), and 3.0 (range $=2.2-4.3, \mathrm{SD}=0.7)$, respectively. The average noise values in the corresponding FBP studies for air, $\mathrm{WM}$, bone, and CSF were 3.4 (range $=1.8-8.4, \mathrm{SD}=1.9$ ), 3.8 $($ range $=3.1-4.7, \mathrm{SD}=0.5), 60.2($ range $=28.9-69.5, \mathrm{SD}=$ 12.5 ), and 4.4 (range $=3.9-5.4, \mathrm{SD}=0.5$ ), respectively. The ASIR studies had statistically significant decreased CSF noise (3.0 versus 4.4, $P=.0000008)$, but no noise differences were seen in air $(P=$ $.46)$, bone $(P=.26)$, or WM $(P=.22)$.

\section{Qualitative Analysis}

There were no significant differences between the ASIR studies and FBP studies with respect to the averaged scores for diagnostic acceptability $(P=.33)$, sharpness $(P=.45)$, or noise $(P=.84)$ (Table). There was a nonsignificant trend that the ASIR studies 

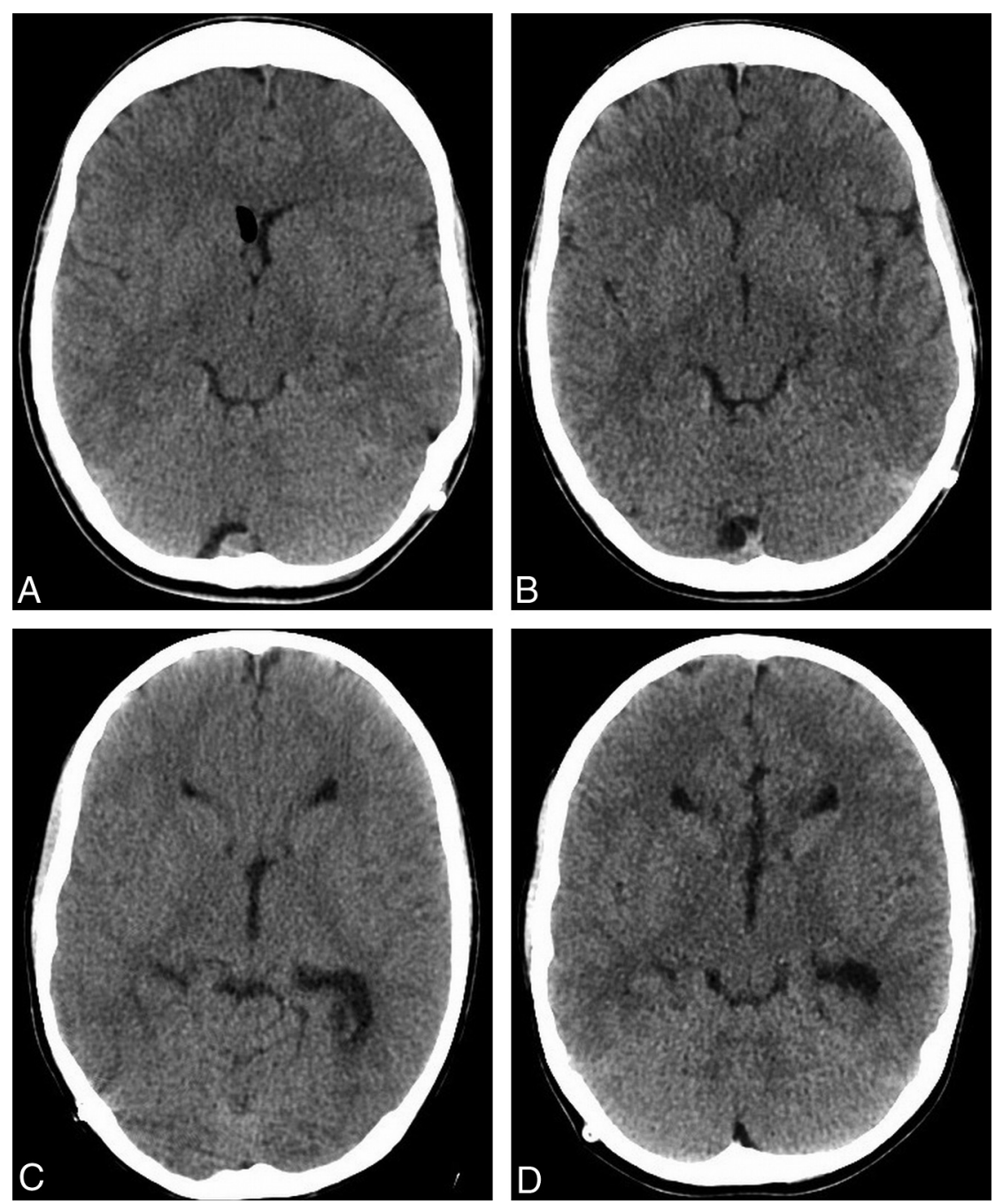

FIG 4. Examples of study patients. Images from $\mathrm{FBP}(A)$ and $A S I R(B)$ examinations performed 26 days apart in a 14-year-old patient, with a $23.7 \%$ decrease in the CTDI (34.9-27.5 mGy) and a $39.6 \%$ decrease in the DLP (581.5-389.3 mGy-cm) in the latter examination. Images from FBP (C) and ASIR $(D)$ examinations performed 77 days apart in a 10 -year-old patient, with a $26.4 \%$ decrease in the CTDI ( 32.3 to $24.7 \mathrm{mGy}$ ) and a $25.8 \%$ decrease in the DLP (452.3 to $349.0 \mathrm{mGy}-\mathrm{cm}$ ) in the latter examination.

had a lower artifacts score ( 1.8 versus $2.1, P=.06)$. No studies were given a score by either reader indicating an uninterpretable examination (ie, a 4 for diagnostic acceptability, a 4 or 5 for image sharpness, a 4 for noise, or a 4 for artifacts). All studies were rated as either a 1 or 2 for diagnostic acceptability. In the FBP studies, there was very good (0.80), perfect (1.00), very good (0.85), and perfect (1.00) agreement for diagnostic acceptability, sharpness, noise, and artifacts, respectively. In the ASIR studies, there was perfect (1.00), good (0.50), perfect (1.00), and perfect (1.00) agreement for diagnostic acceptability, sharpness, noise, and artifacts, respectively.

\section{DISCUSSION}

Multiple different strategies have been adopted in the past in an attempt to lower the cumulative head CT radiation dose, ${ }^{25-28}$ including decreasing the milliamperes, shielding (ie, thyroid shields, bismuth eye shields), automatic tube-current modulation, angling the gantry to exclude the orbits, and so forth. Dosereduction techniques that involve reducing the milliamperes, however, are limited by the resulting increased image noise and decreased CNR when using FBP as the reconstruction algorithm. There has been some interest in creating specific adaptive filters to reduce this image noise (for example the Neuro 3D filter; GE Healthcare), which have been reported to be able to reduce image noise by approximately $30 \% .^{29}$

Our findings of a $22 \%$ reduction in the average $\mathrm{CTDI}_{\mathrm{vol}}$ and a $24 \%$ reduction in the average DLP when using 20\% ASIR, at no significant detriment to subjective image quality, are consistent with the prior published results performed in adults (Fig 4). Kilic et $\mathrm{al}^{21}$ consecutively performed nonenhanced head CT scans in 149 patients; the first 51 patients underwent their original FBP protocol, and the remaining 98 patients underwent their lower-dose IR protocol reconstructed at 30\% ASIR. The group reported a $31 \%$ decrease in the average DLP of their ASIR studies, without a significant change in image quality or interpretability. The study published by Korn et $\mathrm{al}^{22}$ involved 90 adult patients undergoing nonenhanced and enhanced CT of the brain who were randomized to be scanned with either their original head CT FBP protocol or with 1 of 2 low-dose IR protocols. All of these examinations were then subsequently reconstructed with both FBP and IRIS and compared. The authors found that a $15 \%$ dose reduction did not impact subjective or objective image quality when the images were reconstructed with IRIS but that a $30 \%$ dose reduction resulted in noticeable degradation of image quality when reconstructed with IRIS.

The methodology that we used in our phantom analysis is a variation of the phantom work performed by Hara et $\mathrm{al}^{14}$ to help determine their initial ASIR settings for abdominal CT. Unlike Hara et al (who used a Gammex 464 phantom), we used an Alderson-Rando head phantom because it is constructed with radiologically tissue-equivalent material, including bone. ${ }^{30} \mathrm{We}$ then obtained multiple-dose data points at decreasing $10 \% \mathrm{~mA}$ increments relative to our institutional pediatric head CT protocol. Like Hara et al, we reconstructed each scan at increasing 10\% ASIR intervals. The ASIR and milliampere settings that we used for our patient study were chosen on the basis of the phantom study analysis, which demonstrated that at $20 \%$, ASIR examinations performed with $20 \% \mathrm{~mA}$ reductions had noise comparable with that of the full-dose examinations reconstructed with FBP. Although Korn et $\mathrm{al}^{22}$ used IRIS rather than ASIR, our phantom data corroborate their findings that standard image quality is obtainable until about $20 \%$ dose reduction when IR is used. How- 

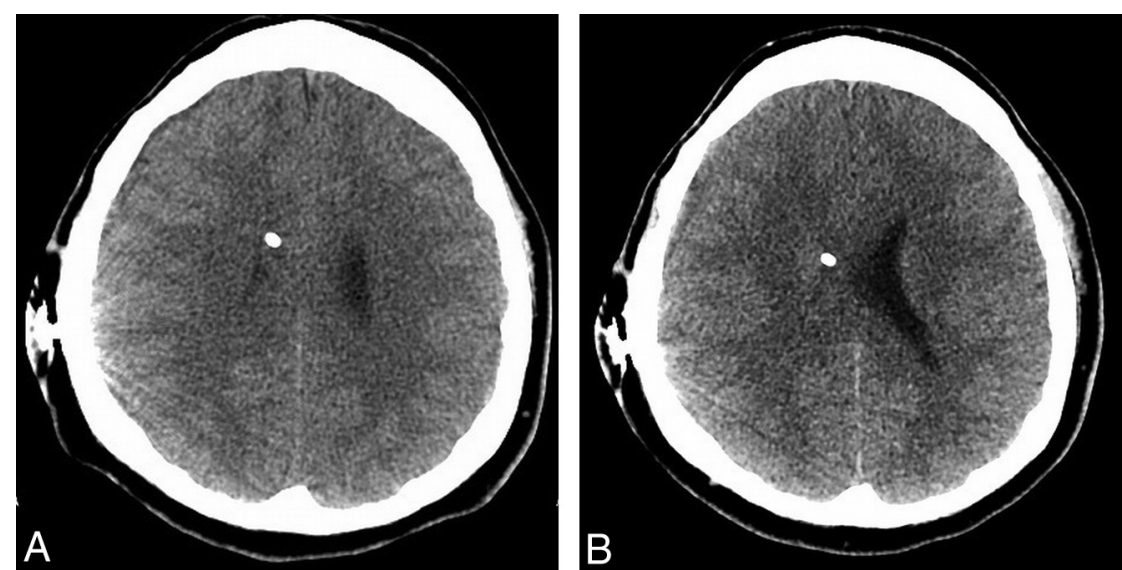

FIG 5. FBP $(A)$ and ASIR $(B)$ images through the level of the shunt reservoir in a 17-year-old boy, in which there are subjectively more reconstruction artifacts around the reservoir in the former study. The 2 examinations were performed 92 days apart, and the latter examination represented a $30.0 \%$ decrease in the CTDI (38.0-26.6 mGy) and a 27.5\% decrease in the DLP (613.8-444.9 mGy-cm).

ever, our phantom data by using the Alderson-Rando phantom suggest that additional radiation dose reduction may be feasible with similar image noise, if the studies are reconstructed with a higher level of ASIR (ie, 30\% dose reduction reconstructed with $30 \%$ ASIR). This could be confirmed with additional phantom and patient studies.

We found that CSF demonstrated significantly decreased noise in our ASIR examinations relative to our FBP examinations, while the other tested densities (WM, bone, air) did not demonstrate any significant noise differences between the 2 groups. This is similar to the findings of Kilic et $\mathrm{al},{ }^{21}$ which they attributed to the propensity of ASIR to disproportionately reduce noise in smoother areas. ${ }^{31}$ We also found that our subjective image scores for image artifacts tended to be lower in our ASIR examinations (Fig 5). This did not reach statistical significance, possibly resulting from our small patient sample. Prakash et al, ${ }^{12}$ however, did not note any improvement in the severity of windmill helical, beam-hardening, or metallic streak artifacts with the application of ASIR in their study comparing 156 patients who underwent abdominal CT reconstructed with ASIR against 66 different patients who underwent abdominal CT with FBP. One reason for these discordant findings could be that, unlike Prakash et al, we were able to directly compare the same reconstruction artifacts in the same patients. The effect of ASIR on reconstruction artifacts caused by high-attenuation objects, potentially relating to its smoothing effect, would be an interesting area of future research.

Our study had a few significant limitations. To begin with, our sample size was very small, with only 12 patients included. Because this was a preliminary study, we first wanted to assess the feasibility of using ASIR in pediatric head CT with concurrent dose reductions before extending the use of ASIR to the larger pool of pediatric patients necessary to perform a more powerful study. Another potential limitation was that 3 of our FBP comparison examinations were performed on our older HighSpeed CT/I scanner, though other publications have also successfully compared FBP and ASIR CT examinations performed on different CT scanners ${ }^{11,13,14,32}$ in a similar manner, even occasionally between different manufacturers. None of our head CT examinations concurrently used automatic tube-current modulation (Au-
tomA/SmartmA; GE Healthcare). Although tube-current modulation has shown promise in reducing pediatric head $\mathrm{CT}$ radiation dose, ${ }^{33}$ we have not yet incorporated tube-current modulation into our routine pediatric head CT examinations. This is partly due to concerns that actual dose reductions may be more modest than originally believed ${ }^{30}$ and that patients could be theoretically overdosed if they are not centered appropriately at the isocenter of the CT gantry. Our phantom study was limited in the sense that we used the head of a small adult Rando phantom, rather than a pediatric anthropomorphic phantom. Unlike the thorax or abdomen (which grows linearly with the child's age), a child's head grows rapidly until approximately 6 years of age, at which point there is a gradual plateau, explaining why our phantom's size allowed us to approximate the size of a 7-year-old child. ${ }^{23}$ Finally, we used small regions of interest $\left(0.5-1.0 \mathrm{~cm}^{2}\right)$ when performing our quantitative analysis, relating to the small areas that we were measuring. We were able, however, to measure consistent areas on the ASIR and FBP examinations in a comparison study set.

\section{CONCLUSIONS}

Our results suggest that an approximate $22 \%$ dose reduction is feasible in nonenhanced pediatric head CT when reconstructed with $20 \%$ ASIR. We were also able to substantiate our ASIR head CT settings with phantom data. Our study may be helpful in promoting the use of ASIR in pediatric head CT to lower radiation exposure, which is even more important than in adults, considering children's increased radiation-induced cancer risk. ${ }^{2,3,6,7}$ Additional research with a larger patient population would be beneficial to further substantiate the potential role of ASIR in pediatric head CT. Future areas of characterization could include the concurrent use of automatic tube-current modulation with ASIR as well as the feasibility of larger radiation dose reductions while maintaining diagnostic image quality.

Disclosures: Ashok Panigrahy_RELATED: Consulting Fee or Honorarium: GE Healthcare, Comments: on Pediatric Medical Advisory Board for GE Healthcare; have also given lectures about CT, UNRELATED: Board Membership: GE Healthcare Pediatric Medical Advisory Board. 


\section{REFERENCES}

1. Mettler F, Thomadsen B, Bhargavan M, et al. Medical radiation exposure in the U.S. in 2006: preliminary results. Health Phys 2008;95:502-07

2. Berrington de Gonzalez A, Mahesh M, Kim K, et al. Projected cancer risks from computed tomography scans performed in the United States in 2007. Arch Intern Med 2009;169:2071-77

3. Strauss K, Goske M, Kaste S, et al. Image gently: ten steps you can take to optimize image quality and lower $\mathrm{CT}$ dose for pediatric patients. AJR Am J Roentgenol 2010;194:868-73

4. Goske M, Applegate K, Bulas D, et al. Image gently: progress and challenges in CT education and advocacy. Pediatr Radiol 2011; 41(suppl 2):461-66

5. Larson D, Johnson L, Schnell B, et al. Rising use of CT in child visits to the emergency department in the United States, 1995-2008. Radiology 2011;259:793-801

6. Karlsson P, Holmberg E, Lundell M, et al. Intracranial tumors after exposure to ionizing radiation during infancy: a pooled analysis of two Swedish cohorts of 28,008 infants with skin hemangioma. $R a-$ diat Res 1998;150:357-64

7. Brenner D, Elliston C, Hall E, et al. Estimated risks of radiationinduced fatal cancer from pediatric CT. AJR Am J Roentgenol 2001;176:289-96

8. Michel M, Jacob S, Roger G, et al. Eye lens radiation exposure and repeated head CT scans: a problem to keep in mind. Eur J Radiol 2011 April 11. [Epub ahead of print]

9. Fleischmann D, Boas F. Computed tomography: old ideas and new technology. Eur Radiol 2011;21:510-17

10. Prakash P, Kalra M, Ackman J, et al. Diffuse lung disease: CT of the chest with adaptive statistical iterative reconstruction technique. Radiology 2010;256:261-69

11. Prakash P, Kalra M, Digumarthy S, et al. Radiation dose reduction with chest computed tomography using adaptive statistical iterative reconstruction: initial experience. $J$ Comput Assist Tomogr 2010;34:40-45

12. Prakash P, Kalra M, Kamboadake A, et al. Reducing abdominal CT radiation dose with adaptive statistical iterative reconstruction technique. Invest Radiol 2010;45:202-10

13. Sagara Y, Hara A, Pavlicek W. Abdominal CT: comparison of lowdose CT with adaptive statistical iterative reconstruction and routine-dose CT with filtered back projection in 53 patients. AJR Am J Roentgenol 2010;195:713-19

14. Hara AK, Paden RG, Silva AG. Iterative reconstruction technique for reducing body radiation dose at CT: feasibility study. AJR Am J Roentgenol 2009;193:764-71

15. Gosling O, Loader R, Venables P. A comparison of radiation dose between state of the art multislice CT coronary angiography with iterative reconstruction, multislice $\mathrm{CT}$ coronary angiography with standard filtered back-projection, and invasive diagnostic coronary angiography. Heart 2010;96:922-26

16. Heilbron BG, Leipsic J. Submillisievert coronary computed tomography angiography using adaptive statistical iterative reconstruction: a new reality. Can J Cardiol 2010;26:35-36

17. Leipsic J, Labounty M, Heilbron B, et al. Adaptive statistical iterative reconstruction: assessment of image noise and image quality in coronary CT angiography. AJR Am J Roentgenol 2010;195:649-54

18. Cornfield D, Isreal G, Detroy E, et al. Impact of Adaptive Statistical
Iterative Reconstruction (ASIR) on radiation dose and imagine quality in aortic dissection studies: a qualitative and quantitative analysis. AJR Am J Roentgenol 2011;196:W336-40

19. Vorona G, Ceschin R, Clayton B, et al. Reducing abdominal CT radiation dose with the adaptive statistical iterative reconstruction technique in children: a feasibility study. Pediar Radiol 2011;41: 1174-82

20. Mieville F, Gundinchet F, Rizzo E, et al. Paediatric cardiac CT examinations: impact of the iterative reconstruction method ASIR on image quality-preliminary findings. Pediatr Radiol 2011;41: $1154-64$

21. Kilic K, Erbas G, Guryildirim M. Lowering the dose in head CT using adaptive statistical iterative reconstruction. AJNR Am J Neuroradiol 2011;32:1578-82

22. Korn A, Fenchel M, Bender B. Iterative reconstruction in head CT: image quality of routine and low-dose protocols in comparison with standard filtered back-projection. AJNR Am J Neuroradiol 2012;33:218-24

23. Kleinman P, Strauss K, Zurakowski, et al. Patient size on CT images as a function of age at a tertiary care children's hospital. $A J R A m J$ Roentgenol 2010;194: 1611-17

24. American College of Radiology. CT Accreditation Phantom Instructions, October 2011. http://www.acr.org/accreditation/computed/ qc_forms/Phantom_Testing_Instruction_Final.aspx. Accessed December 28, 2011

25. Kanal K, Vavilala M, Raelson C, et al. Variation in pediatric head CT imaging protocols in Washington state. J Am Coll Radiol 2011;8: $242-50$

26. Udayasankar U, Braithwaite K, Arvantiti M, et al. Low-dose nonenhanced head CT protocol for follow-up evaluation of children with ventriculoperitoneal shunt: reduction of radiation and effect on image quality. AJNR Am J Neuroradiol 2008;29:802-06

27. Hopper K, Neuman J, King S, et al. Radioprotection to the eye during CT scanning. AJNR Am J Neuroradiol 2001;22:1194-98

28. Smith B, Dillon W, Gould R, et al. Radiation dose-reduction strategies for neuroradiology CT protocols. AJNR Am J Neuroradiol 2008;28:1628-32

29. Toth T, Hsieh J. Strategies to reduce radiation dose in CT; GE Healthcare MDCT scanner strategies to reduce radiation dose. In: Mahesh M. MDCT Physics: The Basics. Philadelphia: Lippincott Williams \& Wilkins; 2009:115-24

30. Papadakis A, Perisinakis K, Oikonomou I, et al. Automatic exposure control in pediatric and adult computed tomography examinations: can we estimate organ and effective dose from mean mAs reduction? Invest Radiol 2011;46:654-62

31. Marin D, Nelson R, Schindera S, et al. Low-tube-voltage, high-tubecurrent multidetector abdominal CT: improved image quality and decreased radiation dose with adaptive statistical iterative reconstruction algorithm-initial clinical experience. Radiology 2010;254:145-53

32. Kambadakone A, Chaudhary N, Desai G, et al. Low-Dose MDCT and CT enterography of patients with Crohn disease: feasibility of adaptive statistical iterative reconstruction. AJR Am J Roentgenol 2011;196:W743-52

33. Smith A, Dillon W, Lau B, et al. Radiation dose reduction strategy for CT protocols: successful implementation in neuroradiology section. Radiology 2008;247:499-506 